\title{
Du travail à la monnaie, essai de perspective sociale de la valeur
}

Examen critique de la vision autoréférentielle de la valeur et de la monnaie

Jean-Marie Harribey

\section{(2) OpenEdition}

Journals

Édition électronique

URL : http://journals.openedition.org/ei/5906

DOI : 10.4000/ei.5906

ISSN : 2553-1891

Éditeur

Association Économie et Institutions

Référence électronique

Jean-Marie Harribey, «Du travail à la monnaie, essai de perspective sociale de la valeur », Économie et institutions [En ligne], 26 | 2017, mis en ligne le 16 septembre 2018, consulté le 19 avril 2019. URL:

http://journals.openedition.org/ei/5906 ; DOI : 10.4000/ei.5906

Ce document a été généré automatiquement le 19 avril 2019

Revue Économie et institutions 


\title{
Du travail à la monnaie, essai de perspective sociale de la valeur
}

\author{
Examen critique de la vision autoréférentielle de la valeur et de la \\ monnaie
}

Jean-Marie Harribey

\section{NOTE DE L'ÉDITEUR}

Une première version de ce texte fut présentée au colloque « Institutionnalismes monétaires francophones : bilan, perspectives et regards internationaux », Lyon, 1-3 juin 2016.

Je remercie les rapporteurs du présent texte pour leurs remarques et suggestions.

1 Le renouveau de la réflexion théorique sur la monnaie doit être relié à l'évolution du capitalisme contemporain depuis le dernier quart $\mathrm{du} \mathrm{xx}^{\mathrm{e}}$ siècle, marquée par l'irruption d'un mode d'accumulation financière qui a abouti à une crise spectaculaire en 2007-2008. $\mathrm{Au}$ cours de cette période, le caractère de bien public de la monnaie est passé au second plan, tandis que celle-ci a été mise au service de la financiarisation de l'économie mondiale qui battait son plein. Les politiques monétaires, soucieuses de contenir l'inflation tout en favorisant le crédit destiné pour l'essentiel aux procédures financières, et les mécanismes de titrisation des titres chaque jour davantage sophistiqués, allaient de pair avec une soumission à des contraintes budgétaires sévères et une austérité salariale toujours plus stricte.

2 Ce processus de quasi-privatisation de la monnaie signifie l'instrumentalisation de son statut d'institution sociale au profit du nouveau rapport travail/capital issu de la globalisation financière. Il se déroule précisément dans la phase où la production de valeur et sa réalisation monétaire entrent dans une crise sans précédent dans l'histoire du capitalisme, car elle conjugue des dimensions multiples, allant du social à l'écologique, de l'économique au financier, du marché aux institutions. 
3 L'objet de ce texte est de confronter les théories analysant les relations entre le travail productif, la valeur, la monnaie et les représentations de ces institutions. Il est construit autour de trois moments. Dans une première partie, nous nous demanderons si considérer que la valeur économique trouve son origine dans les conditions sociales et techniques de production revient chez Marx à attribuer une substance intrinsèque aux objets-marchandises. C'est la thèse défendue par Orléan (2011a) qui voit une similitude sur ce point entre la théorie de la valeur-travail et celle de la valeur-utilité. Il les rejette toutes les deux pour adopter une hypothèse mimétique: les individus élisent un bien comme monnaie parce qu'ils savent que les autres feront le même choix. Cette « élection » exprime la généralité du désir de richesse : la monnaie est élevée au rang de richesse universellement reconnue. Dès lors, la valeur ne serait qu'une représentation symbolique, une pure convention (Théret 2007). Et, en combinant désirs et passions, Lordon (2010) a donné une lecture spinoziste de cette hypothèse qui conduit à l'abandon de toute théorie de la valeur.

4 Nous développerons dans la deuxième partie une perspective différente. Il s'agira de comprendre le fait que, comme le dit Aglietta $(2015,2016)$, la monnaie est un rapport d'appartenance à une communauté politique. Si l'on exclut la version ricardienne de la valeur (au demeurant, en l'absence de monnaie) définie par le contenu en travail « concret », thèse aussi bien rejetée, pour des raisons différentes, par Marx (1867) que par Simmel (1900), il est possible d'articuler dans un corpus théorique cohérent les conditions socio-techniques dans lesquelles le travail est mis en œuvre, la validation sociale par l'échange marchand - donc en monnaie - et les représentations sociales liées à l'état des rapports sociaux. Le travail rendu «abstrait » dans l'échange n'est pas une substance intrinsèque à l'objet-marchandise, il est un caractère spécifique des rapports marchands. Si le mot « substance » est encore employé, il ne peut avoir d'autre sens que "substance sociale ».

Dans la troisième partie, nous montrerons que les représentations sociales de la richesse et de la valeur marquées par des processus mimétiques et/ou autoréférentiels ne peuvent s'étendre à l'infini. Le cas de figure des marchés financiers, souvent pris comme exemples de processus de ce type, est éloquent : un krach financier est toujours un rappel à l'ordre social et productif, aussi bien dans l'esprit de Marx (1894) analysant le capital fictif s'écroulant parce que la valorisation ne pouvait se dispenser du travail, que dans celui de Keynes (1936) théorisant la liquidité, et dans celui des post-keynésiens comme Minsky (1982), Wray (2013) ou Keen (2014) montrant le rôle contradictoire du crédit. Dès lors, le fait que la valeur naisse dans les rapports de production est indissolublement lié au fait que cette valeur se réalise toujours en monnaie, c'est-à-dire doive nécessairement prendre la forme monétaire pour exister. Cette liaison est assurée par la validation sociale. La monnaie pourra ainsi être considérée comme un rapport social à part entière, et pas seulement comme une représentation hors-sol, avec pour corollaire l'idée que la monnaie transformée en capital et en capital seul porte la possibilité d'une crise du capitalisme. Le risque est alors celui redouté par Polanyi (1944) : que la monnaie, après la terre et le travail, soit transformée en marchandise, pour laisser libre cours à «la comédie (in)humaine de l'argent $»^{1}$. 


\section{La monnaie versus la valeur?}

6 Les économistes classiques, essentiellement Smith et Ricardo, avaient rejeté la thèse mercantiliste selon laquelle la richesse correspondait aux métaux précieux, mais ils avaient cru qu'ils pouvaient se dispenser d'intégrer la monnaie à la compréhension de l'« économie réelle " ${ }^{2}$. La "monnaie-voile» fut une des thèses dominant la "science économique " ultérieure, malgré les efforts de Marx et de Keynes pour l'éradiquer. Il faudra attendre la fin du $\mathrm{xx}^{\mathrm{e}}$ siècle pour que la théorie de la monnaie connaisse un renouveau important. Mais, on va le voir, ce n'est pas sans ambiguïtés.

7 La monnaie ne peut pas être véritablement comprise si elle est réduite à son aspect purement économique. Elle n'existe pas non plus seulement dans un cadre capitaliste. Elle est une institution sociale qui dépasse ce cadre. Depuis une trentaine d'années, Michel Aglietta et André Orléan (1982, 1998, 2002), ensemble ou séparément, puis d'autres chercheurs, ont développé une conception originale et féconde de la monnaie. Selon eux, la monnaie n'est pas une conséquence de la multiplication des échanges, elle est une institution sociale précédant ceux-ci et fondée sur une double validation: la confiance partagée par ses utilisateurs et la légitimation politique conférée par la souveraineté qui l'impose. En effet, acceptée par tous, la monnaie est à la fois expression du désir de richesse et lien social.

Il n'est d'économie marchande que monétaire. Nous voulons dire par là que tout rapport marchand, même dans sa forme la plus élémentaire, suppose l'existence préalable de monnaie. Ou bien encore, d'une manière plus concise et plus directe, le rapport marchand est toujours un rapport monétaire. (Aglietta \& Orléan 2002, p. 35$)^{3}$

8 Les auteurs font de cette hypothèse l'axe de leur programme de recherche dont l'ambition n'est pas moins que de «refonder l'économie » (Orléan 2011a) en tant que champ disciplinaire. Il y a fort à faire au moment où tous les dogmes enseignés et assénés connaissent un échec cuisant, à la hauteur de la gravité de la crise systémique du capitalisme mondial, dont on peut dire que son déclenchement doit largement à l'application systématique desdits dogmes, notamment celui qui décrit le modèle concurrentiel comme spontanément efficient et stabilisateur. À la racine de cet échec, explique Orléan, il y a une erreur à la base même de la compréhension de l'économie : toutes les théories de la valeur sont erronées, aussi bien celle qui pense la valeur en termes de travail que celle qui la pense en termes d'utilité. Celles-ci, en apparence opposées, partagent, selon Orléan, une même conception substantialiste qui ferait dépendre la valeur des qualités propres aux objets échangés, le travail d'un côté ou l'utilité de l'autre. Sa thèse est que « la valeur marchande n'est pas une substance [...] qui préexiste aux échanges. Il faut plutôt la considérer comme une création sui generis des rapports marchands, par laquelle la sphère économique accède à une existence séparée, indépendante des autres activités sociales. » (2011a, p. 12). Comment dès lors repenser la valeur? En considérant que «l'élection» d'une monnaie est l'acte fondateur de l'ordre social marchand. «La monnaie est l'institution première des économies marchandes. $L a$ monnaie fonde l'économie marchande.»(2011a, p. 148). Inutile alors de chercher un fondement ou un indicateur de mesure dans le travail comme le fit Marx, ou dans l'utilité et la rareté comme le fit Walras, car « il n'y a d'expression de la valeur que monétaire » (2011a, p. 29). 
9 Si l'on suit ces auteurs, l'alternative théorique à la valeur «substance » doit donc être construite à partir de l'hypothèse mimétique qui postule que les individus élisent un bien comme monnaie parce qu'ils savent que tous, ou une majorité d'entre eux, feront le même choix. Il en résulte que la monnaie est la valeur par excellence, puisqu'elle est par définition parfaitement liquide, dès lors qu'elle est acceptée. La confiance accordée à la monnaie élue est donc la conséquence d'un processus de relations sociales qui interdit de voir les individus agir et s'exprimer au nom d'une rationalité personnelle construite hors de l'environnement social. Cette hypothèse est, selon Orléan, particulièrement vérifiée en ce qui concerne la valeur des actifs financiers, totalement détachée de toute valeur objective dite fondamentale, et qui, en revanche, est le résultat des comportements mimétiques dans lesquels le phénomène des prophéties auto-réalisatrices joue à plein.

Notre objectif est d'examiner si une conception renouvelée de la monnaie autour de l'idée qu'elle est une institution sociale «instituante", et non pas qui résulte des échanges, implique obligatoirement le rejet de toute théorie de la valeur. Cette question est d'autant plus importante qu'elle a quelque rapport avec celle de la définition de la richesse. C'est sans doute aussi l'opinion d'Orléan qui écrit :

On nommera "richesse ", ces biens hypothétiques qui font l'objet d'un désir généralisé de la part des acteurs. [...] Cette analyse se prête volontiers à l'interprétation spinoziste avancée par Frédéric Lordon. Car si le conatus est "l'intérêt à effectuer ses puissances et à les augmenter » comme l'écrit cet auteur, alors, dans l'ordre marchand, il a précisément pour finalité l'appropriation de la richesse. Dans la richesse, c'est l'accès à la généralité qui est recherché par les producteurs-échangistes aux fins d'accroître leur puissance d'être. Il s'ensuit que la lutte des conatus marchands est d'abord une lutte pour la richesse. (Orléan in Lordon 2008, p. 10-11)

11 La généralité du désir de richesse, née " des contraintes sociales marchandes » s'exprime donc par l'«élection » de la monnaie au rang de richesse reconnue par tous et partout. Dès lors, la conclusion d'Orléan est qu'il ne peut y avoir pour cette richesse qu'une définition " autoréférentielle». Dans un de ses articles récents, «La valeur économique comme fait social : la preuve par les évaluations boursières » (Orléan 2015), il entend appliquer la définition d'un fait social de Durkheim : « des manières d'agir, de penser et de sentir, extérieures à l'individu, et qui sont douées d'un pouvoir de coercition en vertu duquel [elles] s'imposent à lui ». L'appliquer à quoi ? À la formation des valeurs boursières, qui constitueraient une «preuve » de la démarche. Très explicitement, Orléan annonce celle-ci comme un prolongement de la thèse qu'il avait soutenue dans L'Empire de la valeur (2011a). Nous rejoignons pleinement l'idée que la valeur est un fait social et non pas le fruit de décisions individuelles, mais le modèle que développe Orléan laisse de côté la discussion contradictoire à laquelle son livre avait donné lieu .

12 Quel est ce modèle ? Il y a d'abord un présupposé : la valeur économique relève de la même épistémologie que les valeurs philosophiques ou religieuses.

[...] les valeurs sont, non pas des mesures, mais bien des forces qui font agir les hommes en leur donnant des raisons de vivre et des buts à atteindre. Selon cette approche, les valeurs sont à l'origine de l'énergie qui fait fonctionner la société. C'est l'influence qu'elles exercent qui pousse les hommes à se dépasser, à s'élever au-dessus d'eux-mêmes [...]; raison pour laquelle on ne peut s'en tenir à l'hypothèse d'individualités fixées une fois pour toutes. Cette approche, qui trouve aisément à s'illustrer du côté des valeurs religieuses ou morales, s'applique aussi bien à la valeur économique. (Orléan 2015) 
13 Ce présupposé est-il tenable ? Peut-être, s’il y avait commensurabilité des valeurs et de la valeur. Est-ce le cas ? Nous ne le pensons pas.

Que se passe-t-il sur les marchés financiers selon Orléan?

À contre-courant des automatismes les plus ancrés de l'analyse économique néoclassique, qui toujours construit le prix par agrégation en partant des estimations privées, il s'agit de jeter sur les faits boursiers une autre lumière afin de faire émerger une manière nouvelle de les comprendre. [...] Les estimations individuelles se construisent à partir de la convention financière, c'est-à-dire à partir du marché lui-même, et non l'inverse. C'est le marché financier qui informe les investisseurs et non le contraire. La valeur, sous la forme du prix, est d'emblée collective. Avec cette conception alternative, l'acteur n'est plus le souverain qui détermine les prix. Tout au contraire, il est agi par le marché. Cette causalité « inversée » est particulièrement visible lors des épisodes de bulles. (Idem)

Dans cet article, Orléan abandonne - ou laisse de côté - l'amalgame qu'il faisait entre la théorie de la valeur de Marx et celle des néoclassiques. Il ne réfute ici que cette dernière, mais tout en gardant la coupure entre la sphère financière et le système productif et ses rapports sociaux. Or, en quoi le refus de considérer les évaluations individuelles conduisant à des prix de marché dément-il la nécessité de regarder ce qu'il se passe dans le système productif?

Par quel mystère l'imitation à la hausse des prix boursiers se transforme-t-elle (souvent brutalement) en imitation à la baisse ? Orléan propose la réponse suivante :

Cependant, lorsque le poids de l'imitation passe un certain seuil critique, se produit un changement qualitatif dans le comportement collectif, à savoir que les deux opinions cessent d'être réparties à égalité ; une des deux opinions - a priori n'importe laquelle - l'emporte sur l'autre. Autrement dit, ce modèle nous donne à voir l'émergence spontanée d'une majorité au sein d'une population qui est pourtant sans convictions personnelles tranchées! Tel est le résultat fondamental qui ici nous intéresse. Il en est ainsi parce que, localement, les choix des voisins ne se répartissent pas nécessairement de façon strictement égale entre les deux options, même si chacun d'entre eux fait un choix purement équiprobable. Cela tient à la nature aléatoire des choix. [...] Pour qu'il en soit ainsi, il importe cependant que l'intensité des interrelations dépasse un certain seuil. En dessous de ce seuil, le fonctionnement global du groupe ou du marché ne s'éloigne pas de la simple somme des préférences individuelles. (Idem)

Le modèle d'Orléan permet-il de savoir vraiment pourquoi l'imitation «passe un seuil critique » et à quel niveau se fixe ce seuil ? Orléan observe les entreprises des nouvelles technologies et constate que, dans la période de la bulle internet, la valorisation boursière atteignait des sommets astronomiques, sans rapport avec les profits réels des entreprises. Soit! Et alors? La bulle ne s'est-elle pas effondrée parce que justement cet écart était intenable? On pourrait encore formuler le problème non résolu autrement: le modèle d'Orléan vise-t-il à expliquer les variations du niveau des prix des actifs financiers et, audelà, celles du niveau des prix des marchandises en général, ou bien à expliquer leur niveau lui-même ${ }^{5}$ Pour le dire très simplement, par exemple, est-ce le fait monétaire qui, en lui-même, explique qu'un avion Airbus vaille 500 millions d'euros, un sous-marin 2 milliards d'euros et une douzaine d'œufs 4 euros ? Répondre à cette question supposera dans un instant de revenir à l'examen des conditions matérielles - sociales et techniques - de production, sans pour autant renoncer à l'idée que la valeur est toujours sous forme monétaire. 
es, comme le montre Orléan, la valeur ne résulte pas d'appréciations individuelles. Mais elle ne résulte pas plus d'un marché financier qui agirait sur les individus, sans que ce marché ne soit lui-même le produit d'un système socio-économique et non celui de simples représentations hors-sol. Il est à craindre que nous n'ayons plus de théorie du profit ni de l'accumulation si la «valeur pour l'actionnaire " résulte seulement de processus mimétiques autoréférentiels. L'économiste post-keynésien Hyman Minsky pose ce problème de la manière suivante :

L'un des vecteurs à travers lesquels les profits affectent l'investissement est le prix des actions ordinaires échangées sur les places boursières. En période faste, le bienêtre de l'actionnariat s'améliore parce que les dividendes distribués aux détenteurs d'actions augmentent et que les prix des actions s'élèvent pour refléter tant l'augmentation des gains que les perspectives optimistes. Cette augmentation de la richesse des actionnaires génère une consommation accrue de la part des bénéficiaires de dividendes, qui crée à son tour une nouvelle augmentation des profits. Cette relation entre les profits et la consommation financée par les revenus des profits est un des facteurs de l'instabilité haussière ${ }^{6}$. (Minsky Hyman 2016, p. 354)

Aussi, pour s'éloigner de l'idée que toute théorie de la valeur doit être abandonnée, et donc que la théorie de la monnaie remplacerait la théorie de la valeur, il convient d'examiner le rapport entre valeur et monnaie.

\section{La monnaie, rapport social}

La valeur, dont l'expression ne peut être qu'en monnaie, a-t-elle un fondement au-delà (ou en deçà) de son expression? Dans son dernier ouvrage qui propose une synthèse de quarante années de recherche sur la monnaie, La Monnaie entre dettes et souveraineté, Michel Aglietta aborde la question ainsi : "L'échange contre monnaie, c'est-à-dire le paiement, est l'opérateur de la valeur », ce qui signifie que :

Tout échange est enregistré en partie double dans les comptes de chacun des deux échangistes comme un flux d'objets marchands ayant pour contrepartie un flux de monnaie. Cette inscription en partie double ne signifie pas que deux valeurs préexistantes aient été égalisées, mais qu'une valeur d'objets marchands a été créée et validée par un flux de monnaie. C'est en ce sens que la monnaie est l'opérateur de la valeur. (Aglietta 2016, p. 46)

21 On ne pourrait douter alors que, à cette étape, la monnaie a créé selon lui la valeur contre laquelle elle s'échange. Pourtant, est-ce la même chose que de dire «c'est par la logique de la monnaie que se reconnaît et que s'institue la valeur»? (2016, p. 46). La reconnaissance porterait-elle sur un déjà là, sur un potentiel déjà là ? Le doute commence à s'installer. «La valeur d'usage est distincte de la valeur comme le signifié est distinct du signifiant dans le langage. [...] Il y a donc dans le paiement qui institue la valeur, comme dans le langage, un détachement entre le signifiant (la monnaie) et le signifié (l'objet marchand).» (Ibid., p. 45)

22 La conclusion d'Aglietta se précise peu à peu : «La monnaie institue la valeur sociale par équivalence. L'échange d'équivalents présuppose l'existence d'un espace homogène de commensurabilité où les actes sociaux sont des objets d'évaluation sous un standard commun de mesure.» (2016, p. 83) «La monnaie agit comme le langage, elle rend commensurables les valeurs et dettes car celles-ci sont évaluées avec une unité de compte unique et censée agir comme un étalon de mesure invariable.» (2016, p. 93) Mais on était 
parti avec l'idée que des valeurs préexistantes n'avaient pas été égalisées, et on arrive avec l'idée contraire. Pour notre part, en termes simples, nous disons que l'instrument de mesure (la monnaie) ne doit pas être confondu avec l'objet de la mesure : le mètre-étalon ne détermine pas si un individu est grand ou petit.

La conclusion d'Aglietta est alors quelque peu en décalage par rapport à l'hypothèse de départ :

La valeur conférée par l'opérateur monétaire est le sens commun des choses qui deviennent des marchandises. La marchandise est une chose privée dans la production qui doit rencontrer le désir d'autrui pour devenir un objet de consommation. Elle porte donc une tension entre le privé et le social qui résulte de la séparation des activités humaines dans la société. La monnaie réunit ce qui est séparé. En permettant d'assouvir le désir d'autrui, elle valide l'activité du producteur de l'objet dans le paiement, lui donnant une reconnaissance sociale. (Aglietta 2016, p. 45, souligné par nous).

24 C'était d'ailleurs ce qui ressortait déjà implicitement dans son ouvrage fondateur de l'École de la régulation (1997, p. 55, souligné par nous) où le concept de valeur « exprime les rapports par lesquels le travail particulier, accompli dans les différents lieux où sont rassemblées les forces productives, devient travail social ». Ce qui est une manière de réexprimer la métaphore de Marx du 《saut périlleux $»^{7}$ que doit accomplir la marchandise pour que le travail soit socialement validé.

Si l'on n'a pas peur des mots, l'activité du producteur dont parle Aglietta, c'est son travail, entendu comme englobant, outre son exécution, l'ensemble des conditions techniques et sociales de celui-ci. Cette conclusion empêche donc, à notre sens, de considérer la théorie du mimétisme comme pouvant se substituer à la théorie de la valeur de Marx, si on a pris soin d'intégrer à cette dernière la notion de validation sociale, et ce en suivant Aglietta lui-même. Dans ce cadre, la théorie du mimétisme serait moins heuristique que celle de Marx, dès lors que l'ensemble des phénomènes productifs (à la fois sociaux et techniques) seraient séparés de leurs représentations. Si cette séparation perdurait, elle perdrait tout caractère scientifique.

Une autre manière d'interroger la nature sociale de la monnaie se trouve dans la lecture spinoziste qu'a proposée Frédéric Lordon. On l'a dit, la monnaie est une institution sociale fondée sur la confiance partagée, née du fait qu'elle est désirée par tous parce que tous la désirent, et sur la légitimation politique conférée par la souveraineté qui l'impose. En ce sens, la monnaie n'est pas simplement un instrument d'échange, elle est un rapport social à part entière. Plus exactement, dit Lordon, «la monnaie [est] le nom d'un certain rapport social et [...] l'argent est le nom du désir qui prend naissance sous ce rapport » (2010, p. 27).

27 Dès lors, Lordon rejoint Orléan pour dire qu'il n'y a plus besoin de théorie de la valeur, car toutes les théories de la valeur sont peu ou prou substantialistes. On comprend ainsi que la dilution des classes sociales trouve chez Lordon un substrat théorique dans la substitution de l'« exploitation passionnelle » (2010, P. 148 et suiv.) au prélèvement de la plus-value. La notion de plus-value est récusée parce qu'elle renvoie à « une théorie substantialiste de la valeur - dont la substance est ici le temps de travail abstrait» (2010, p. 148). En suivant Spinoza, il écrit : « la valeur ni le sens n'appartiennent aux choses mais sont produits par les forces désirantes qui s'en saisissent» (2010, p. 90, souligné par l'auteur). Et il cite le philosophe :

Nous ne nous efforçons pas vers quelque objet, nous ne le voulons pas, nous ne le poursuivons, ni ne le désirons parce qu'il est un bien, mais au contraire nous ne 
jugeons qu'un objet est un bien que parce que nous le voulons, le poursuivons et le désirons. (Spinoza 2008 [1677], Éthique III, 9, scolie, in Lordon 2010 p. 90, 149 et 212) nos appréhensions spontanées, que ce n'est pas tant la valeur, préexistante et objectivement établie, qui attire à elle le désir que le désir qui, investissant les objets, les constitue en valeur» (2010, p. 149). Condillac, l'un des introducteurs, avec Voltaire, de Locke en France et théoricien des sensations comme source de la connaissance, n'aura plus qu'à transformer le précepte de Spinoza en aphorisme qui servira de base, non pas à l'abandon de toute théorie de la valeur, mais à la théorie néoclassique de la valeur-utilité : «Une chose n'a pas de valeur parce qu'elle coûte, comme on le suppose ; mais elle coûte parce qu'elle a une valeur. $»^{8}$ La formulation de Lordon sur le rôle des affects chez Spinoza rejoindrait donc celle sur les sensations chez Condillac. Matthieu Montalban voit une seule différence entre la théorie de la valeur-utilité et celle de Lordon issue de Spinoza: «au lieu de considérer les fonctions de préférence comme exogènes et les agents rationnels, les préférences sont ici endogènes car construites par la composition des affects, les coutumes et les différentes institutions de valorisation et de définition de la qualité » (2012).

Comment éviter alors le mirage de la valeur-utilité, dont la première conséquence, sinon la raison d'être profonde, est d'évacuer de l'analyse les conditions socio-techniques de production, et donc les rapports sociaux ? Une fois de plus, il faut reconnaître le mérite de l'économie politique, en rupture avec cette conception, d'avoir renoué avec l'intuition d'Aristote $(1992,1993)$ et réhabilité la distinction entre valeur d'usage et valeur d'échange, tandis que Condillac, Say et, à leur suite, ladite science économique moderne défendront constamment que l'utilité et la valeur ne font qu'un, et feront même disparaître les concepts de valeur d'usage et de valeur d'échange. Avec la valeur réduite aux désirs, cela risque d'être difficile de marquer la frontière avec la toute nouvelle neuro-économie behaviouriste que par ailleurs Lordon (2010/2017) fustige avec beaucoup d'arguments.

Puisque, selon Spinoza, nous allons à l'objet du désir et qu'ainsi il devient bon à nos yeux, pourquoi y allons-nous ? Et puisque, selon Lordon, c'est le désir qui le fait devenir valeur, quelle est la source de ce désir qui doit s'insérer obligatoirement, nous dit Spinoza, dans une succession de causes et d'effets? Si l'on se reporte à l'intégralité de la scolie de Spinoza que cite Lordon, on peut y lire :

Cet effort, quand il se rapporte à l'Âme seule, est appelé Volonté; mais, quand il se rapporte à la fois à l'Âme et au Corps, est appelé Appétit ; l'appétit n'est par là rien d'autre que l'essence même de l'homme, de la nature de laquelle suit nécessairement ce qui sert à sa conservation; et l'homme est ainsi déterminé à le faire. De plus, il n'y a nulle différence entre l'Appétit et le Désir, sinon que le Désir se rapporte généralement aux hommes, en tant qu'ils ont conscience de leurs appétits, et peut pour cette raison, se définir ainsi: le Désir est l'appétit avec conscience de lui-même. » (Spinoza, 2008 [1677], p. 260-261)

31 Arrivés à ce point, nous sommes inclus, selon la lecture spinoziste, dans une chaine déterministe absolue : ce que nous estimons être libre arbitre n'est que la manifestation de l'ignorance des causes qui nous font agir, hormis la tristesse ou la joie, qui, pour la première, nous pousse à résister ou qui, pour la seconde, nous fait agir pour la perpétuer ; dans les deux cas, notre puissance d'agir se trouve augmentée. Comment se fait-il alors que, grâce à la résistance - d'autant plus forte que la tristesse est grande ${ }^{9}$ - ou grâce à la 
joie qui nous fait agir pour rester joyeux, nous ne soyons pas toujours joyeux, ne baignions pas en permanence dans la félicité et restions le plus souvent asservis?

Bref, il y a là, à notre sens, un échafaudage théorique encore mal assuré. Au lieu d'inverser le rapport de la valeur et du désir, comme le propose Lordon, ne s'agirait-il pas plutôt de sortir de ce dilemme pour considérer qu'il y a deux réalités dont la rencontre va valider leur reconnaissance simultanée : d'un côté, le désir nous pousse à vouloir la valeur, de l'autre, la formation de la valeur reflète les conditions socio-techniques de production de l'objet $d u$ désir. Autrement dit, contrairement à tout l'enseignement standard de l'économie, contrairement aussi à l'implicite de la thèse néo-institutionnaliste sur la monnaie et la valeur, la théorie dite de la valeur-travail au sens de Marx (mais pas de Ricardo) est incluse dans l'âpreté du désir, l'utilité et la rareté, que cette dernière soit réelle ou fantasmée : parce que le désir est là, l'homme va produire, mais les conditions sociales et techniques de cette production, qui dépassent l'expérience personnelle de chaque individu, n'ont pas grand-chose à voir avec des affects préalables tristes ou joyeux. Et cela nous semble parfaitement compatible avec l'expression de Lordon citée plus haut: «la valeur ni le sens n'appartiennent aux choses mais sont produits par les forces désirantes qui s'en saisissent ».

Un grand nombre de critiques ont été émises à l'encontre de la loi de la valeur issue des classiques anglais et de Marx : rejeter toute idée de substance est leur point commun. Le problème est que si vous chassez la substance du travail, en surgit une autre en tout point semblable, chez Lordon lui-même : "Avant même la conversion du produit en argent, le patron capitaliste capte la même chose que n'importe quel autre patron spécifique (mandarin, croisé, chorégraphe...), l'objet princeps de capture du patron général: de l'effort, c'est-à-dire de la puissance d'agir » (2010, p. 156). Mais qu'est-ce que cet effort, cette puissance d'agir, sinon la force de travail de Marx, convertie en travail abstrait face au patron capitaliste générique de Lordon?

À partir de la fin du xix siècle, le refus de la théorie de la valeur de Marx fut théorisé par les auteurs marginalistes qui fondèrent la valeur sur la comparaison des utilités marginales des biens, parce que l'utilité marginale décroît au fur et à mesure que les désirs sont satisfaits. Mais comment appliquer cela au désir de possession de l'argent, dont on voit bien qu'il vérifierait l'axiome inverse : plus on en possède, plus on en veut? Seul cet axiome inverse permet de saisir « l'esprit du capitalisme », son idéaltype au sens où l'entend Max Weber (1905), et la soif inextinguible d'accumulation. Et nous prenons le mot «soif » à dessein, puisque la métaphore de l'eau dans le désert sert de point de départ aux premiers économistes marginalistes. Comme le raisonnement ne s'applique pas à la monnaie-argent, la métaphore de l'eau n'ayant pas de portée générale, et qu'il est donc invalidé (réfuté, dirait Popper [1995]), il ne reste plus qu'une seule échappatoire: évacuer de l'analyse l'objet récalcitrant, qui n'entre pas dans le modèle, la monnaie. Or, Walras construit son modèle d'équilibre général en posant une première équation où il considère la monnaie comme un autre bien, et il anéantit du même coup la pertinence de son modèle. La théorie néoclassique part du désir et postule que la consommation rapproche du point de satiété. Ce faisant, elle méconnait totalement le désir, toujours prêt à renaître, jusqu'à la mort, tel celui de Don Juan pour qui la possession d'une femme crée le désir d'en posséder immédiatement une autre. 


\section{La monnaie et la valeur : pas l'une sans l'autre, elles sont un rapport social}

Revenons au processus instituant la monnaie. Le choix d'un bien comme monnaie résultet-il seulement d'une imitation des individus entre eux ? La monnaie est élue équivalent universel parce qu'elle est garantie par la puissance publique, et elle n'est richesse par excellence que si, parallèlement, un travail productif est effectué. Autrement dit, comme la monnaie est un droit à valoir sur la production, il faut considérer que notre économie n'est pas une économie monétaire mais une économie monétaire de production, ainsi que l'ont exprimé avec des mots différents Marx et Keynes. En abandonnant toute théorie de la valeur fondée sur le travail, on ne peut voir dans les excès de la finance qu'un phénomène autoréférentiel qui s'entretiendrait de lui-même sans aucun lien avec ce qui se déroule dans la production. Cette croyance empêche de voir la crise globale actuelle comme une crise de l'ordre social imposé par le capital dont la suraccumulation est une tendance revenant régulièrement. $\mathrm{Et}$, au sujet des retraites, beaucoup croyaient que la finance était capable de transférer dans le temps des richesses réelles. Or, la finance est seulement capable de transférer dans le temps la propriété des richesses, si celles-ci sont produites et si, bien sûr, elles ne sont pas fictives. On pourrait dire que cette dernière remarque relève du simple bon sens, mais elle traduit une idée complexe et subtile de Marx qui met en relation dialectique les surplus-surtravail (on est au niveau de la substance) et le type de rapports sociaux qui permet à une classe de s'en emparer sous la forme valeur, sous-entendu monétaire. En d'autres termes, au binôme de l'économie politique classique (tant chez Smith que chez Ricardo) valeur d'usage/valeur d'échange, Marx oppose le triptyque suivant : la valeur d'usage est une condition de la valeur en tant que fraction du travail socialement validé monétairement, laquelle apparaît dans l'échange par le biais d'une proportion, la valeur d'échange qui, en tendance, est mesurée par l'équivalent monétaire de la quantité de travail nécessaire en moyenne dans la société considérée, une fois satisfaite l'exigence d'un taux moyen de profit pour le capital. Apparaît donc nettement ici que le cœur de la discussion est le concept de validation, celle-ci étant, pourrait-on dire, le point de passage du travail à la valeur monétaire.

En définitive, est-il aussi illégitime que le pensent certains théoriciens institutionnalistes de la monnaie de concevoir une théorie de la valeur en tant qu'expression des rapports sociaux ? À notre sens, on ne peut pas glisser du constat que le marché crée la liquidité à l'idée que le marché crée la valeur financière. Beaucoup d'auteurs oscillent entre deux thèses qui nous paraissent incompatibles : d'une part, celle d'un détachement vis-à-vis de toute référence à l'économie productive, d'autre part, celle pour laquelle l'efficacité et la rentabilité du système productif restent centrales de manière sous-jacente à moyen et long terme. Notre problématique va donc à l'encontre de celle que proposait Orléan dans un écrit ancien (1999, p. 183) : «On retrouve ici le caractère clos du monde de la finance qui détermine ce que sont les fondamentaux à partir des interprétations qu'il produit. Les valeurs fondamentales n'existent pas en elles-mêmes. Il n'y a que des interprétations. »

On mesure alors le risque d'une définition de la richesse par la monnaie et réciproquement celle de la monnaie comme la richesse universellement reconnue, sans référence à la valeur, ce qui fait dire avec à propos à Montalban (2012) que «d'une certaine façon, Aglietta et Orléan (2002) retrouvent une position mercantiliste et, sous un certain angle, keynésienne, mais pas totalement puisque Keynes lui-même n'était pas loin 
d'adhérer à la théorie de la valeur-travail ». En effet, selon nous, le point ultime du raisonnement sur l'autonomie de la finance est que non seulement les fondamentaux ne déterminent plus les valeurs financières, mais que la relation est inversée : ce sont les croyances qui façonneraient les fondamentaux, et la détermination des croyances ellesmêmes serait renvoyée dans un monde éthéré. Or, ce monde est un monde enchanté, fétichisé, parce que le capital y aurait la capacité de « se » valoriser tout seul : «[...] si l'on doit reconnaître que le capital est la source créatrice de toute valeur, aucun capital en particulier ne possède en lui-même la capacité de l'affirmer. Son aptitude à créer de l'utilité sociale, autrement dit sa valeur sociale, seul le marché en juge. " [Orléan, 1999, p. 245-246]. Dès lors, la finance «travaille à la reconnaissance du capital comme source ultime de la valeur et du marché comme son évaluation pertinente » (p. 249).

Le fait que le taux de rendement du capital, ou rentabilité financière, soit devenu une exigence a priori a pour conséquence de faire s'aligner la rentabilité économique sur la première, comme une sorte d'obligation de résultat qui s'exprime vis-à-vis de l'entreprise, laquelle n'a plus d'autre choix que de se retourner contre ses salariés. Le risque est donc assumé finalement par ces derniers. La « déconnexion entre les niveaux de risque et le rendement» (Orléan, 1999, p. 159) pour les détenteurs de capitaux est indéniable, mais elle ne doit pas selon nous être assimilée à une déconnexion entre l'obtention de valeur pour eux et l'activité productive. Si Orléan a raison de dire qu'une crise financière est « un processus de destruction de la liquidité financière » (Orléan, 1999, p. 139, souligné par nous), il a tort d'affirmer qu'il s'agit d'une disparition de «richesse financière » (p. 98, souligné par nous), ou bien qu'« entre son point le plus haut en 2007 et son point le plus bas en 2009, la moitié de la capitalisation boursière s'est volatilisée, soit une perte de 25000 milliards de dollars. C'est assurément une destruction colossale de valeurs, l'équivalent de la moitié des richesses produites en un an sur la planète ou dix fois le produit intérieur brut de la France » (Orléan, 2012) ${ }^{10}$. Or, ce qui s'évanouit selon nous, lorsqu'éclate la bulle, ce n'est pas de la richesse "massivement créée par le marché » comme le dit Orléan (2011a, p. 302), ni même de la valeur, c'est le grossissement précédent du « capital fictif » (Marx 1968, Livre III). Et on peut avancer l'hypothèse que, au-delà des apparences ou des phénomènes purement mimétiques, la crise de l'accumulation financière n'est que l'expression exacerbée de la difficulté à produire et à réaliser de la valeur. Dit encore autrement, il n'y a pas, sur le plan macroéconomique et à long terme, de plus-value financière sans plus-value réelle. Au vu de la crise actuelle, il nous semble d'ailleurs qu'Aglietta (2009) a formulé un jugement s'écartant nettement de l'euphorie affichée par les idéologues des marchés financiers : «Marx a une analyse de l'argent extraordinaire ».

Ce côté « extraordinaire » tient à l'intégration du fait monétaire dans la dynamique du capital, conçu comme un rapport social. Il ouvre la voie aux recherches anthropologiques qui viendront plus tard. Ainsi, en suivant Marcel Mauss (1923-1924), la monnaie est un «fait social total », car elle est inséparable du triptyque donner-recevoir-rendre ${ }^{11}$, et parce qu'elle est constitutive de la dette comme relation sociale la plus ancienne et la plus répandue des sociétés.

Comme la monnaie possède deux faces, privée et sociale, elle ne peut être séparée de la valeur. Elle éteint la dette économique et elle transmet aussi les dettes sociales qui n'ont pas vocation à s'éteindre, et pas seulement dans les sociétés dites primitives. La monnaie a donc une double dimension. D'une part, elle est un bien privé, un instrument d'accumulation reflétant des rapports sociaux dès l'instant où sa possession en quantité 
suffisante permet l'achat de la force de travail qui transforme l'argent en capital en perpétuel accroissement. Il s'ensuit que, si «la monnaie n'est pas un bien particulier soumis à la loi de la valeur » (Aglietta \& Cartelier 1998, p. 133), elle n'élimine pas la valeur en tant que rapport social au sein duquel la force de travail engendre sa possibilité qui se réalisera sur le marché. Pour le dire autrement, distinguer monnaie et argent au sein du capitalisme signifie que la transformation de l'argent en capital (en tant que processus historique et en tant que condition générique du capital) suppose le rapport social d'exploitation de la force de travail.

41 Mais, d'autre part, la monnaie est aussi un bien public parce qu'elle est instituée par la société qui en garantit la validité sur un territoire donné, sans quoi les échanges privés ne pourraient se dérouler, et parce que, grâce à elle, une partie de la richesse peut être socialisée dans les canaux des services non marchands et de la protection sociale. C'est donc par le biais de la monnaie que sont validés les travaux effectués dans la société : que ce soit sur le marché pour le travail productif de marchandises ou sur décision politique pour le travail productif de services non marchands. Cela signifie que la monnaie est l' institution sociale sans laquelle la vente sur le marché de la marchandise ne pourrait avoir lieu, c'est-à-dire que la valeur ne serait pas validée en même temps que l'anticipation capitaliste, et sans laquelle également l'anticipation et la validation conjointes de la satisfaction de besoins collectifs ne pourraient être inaugurées. C'est en ce sens que l'on peut soutenir que le travail employé pour produire des services non marchands (mais monétaires) est productif de valeur ${ }^{12}$.

Le caractère de bien public de la monnaie est invisible dans la conception standard de la monnaie, parce qu'il révèle le conflit de classes pour le partage de la valeur et parce qu'il porte en lui la potentialité d'une société en partie non marchande et donc solidaire. C'est ce qui fonde la légitimité de l'endettement public lorsqu'il s'agit de financer la production d'une richesse collective sous forme d'éducation, de santé, de recherche ou d'infrastructures publiques. Dans la société, la monnaie est un opérateur social d'homogénéisation de tous les travaux. C'est déjà vrai au sein d'un secteur capitaliste pur, c'est également vrai là où un secteur monétaire non marchand coexiste à côté du secteur capitaliste.

Finalement, un point commun rassemble toutes les thèses discutées ici : la valeur est un rapport social (c'est l'enseignement premier de Marx) et la monnaie aussi (c'est l'apport des institutionnalistes prolongeant les intuitions de Keynes $\left.{ }^{13}\right)$. Le problème nait lorsqu'il s'agit de dire ce qu'est un rapport social. S'agit-il d'une représentation hors de tout enracinement objectif, et même d'une représentation créant le réel? Ou bien s'agit-il d'une dialectique entre les rapports de production et les représentations de ceux-ci ? Nous privilégions la seconde hypothèse.

\section{BIBLIOGRAPHIE}

Aglietta Michel (1997) [1976], Régulation et crises du capitalisme, Paris, Odile Jacob. 
Aglietta Michel (2009), « Marx a une analyse de l'argent extraordinaire », Le Point, Hors-série «Grandes biographies, Marx », $\mathrm{n}^{\circ} 3$.

Aglietta Michel (2015), « La monnaie est un rapport social », Entretien avec Michel Aglietta, Les Possibles, $\mathrm{n}^{\circ}$ 6, Printemps 2015 ; https://france.attac.org/nos-publications/les-possibles/ numero-6-printemps-2015/dossier-monnaie-et-finance/article/la-monnaie-est-un-rapportsocial

Aglietta Michel (2016), en collaboration avec Pepita Ould Ahmed et Jean-François Ponsot, La Monnaie entre dettes et souveraineté, Paris, Odile Jacob.

Aglietta Michel \& Cartelier Jean (1998), « Ordre monétaire des économies de marché », in Aglietta Michel \& Orléan André (dir.) , La Monnaie souveraine, Paris, Odile Jacob, p. 128-158.

Aglietta Michel \& Orléan André (1982), La Violence de la monnaie, Paris, Puf.

Aglietta Michel \& Orléan André (dir.) (1998), La Monnaie souveraine, Paris, Odile Jacob.

Aglietta Michel \& Orléan André (2002), La Monnaie entre violence et confiance, Paris, Odile Jacob.

Aristote (1992), Éthique de Nicomaque, Paris, GF-Flammarion.

Aristote (1993), Les Politiques, Paris, GF-Flammarion.

Artous Antoine (2003), Travail et émancipation sociale, Marx et le travail, Paris, Syllepse.

Artous Antoine (2013), « Jean-Marie Harribey, la "sphère non marchande" et la théorie de la valeur de Marx », Contretemps ; http://www.contretemps.eu/lectures/jean-marie-harribey-»sphere-non- marchande- »-theorie-valeur-marx

Artous Antoine (2014), « À nouveau sur Jean-Marie Harribey, la "sphère non marchande" et la théorie de la valeur de Marx ", Contretemps ; https://www.contretemps.eu/a-nouveau-sur-j-mharribey-la-sphere-non-marchande-et-la-theorie-de-la-valeur-de-marx

Condillac Étienne Bonnot de (1776), Le Commerce et le gouvernement considérés relativement l'un à l'autre. Voir https://fr.wikisource.org/wiki/

Le_Commerce_et_le_gouvernement_considérés_relativement_l'un_à_l'autre/Première_Partie/ Section_1

Cordonnier Laurent (2012), « Une théorie de la valorisation? », Revue française de socio-économie, $\mathrm{n}$ - 10, 2012/2, p. 289-294 ; https://www.cairn.info/revue-francaise-de-socio-economie-2012-2page-289.htm

Darmangeat Christophe (2016), Le Profit déchiffré, Trois essais d'économie marxiste, Montreuil, Éditions La ville brûle.

De Brunhoff Suzanne (1967), La Monnaie chez Marx, Paris, Éditions sociales, $3^{\text {e }}$ édition.

Harribey Jean-Marie (2011a), « Frédéric Lordon, Capitalisme, désir et servitude. Marx et Spinoza, La Fabrique, 2010. », Revue de la régulation [En ligne], 9 | 1er semestre / Spring 2011, mis en ligne le 06 juin 2011, consulté le 28 mars 2018. URL : http://journals.openedition.org/regulation/9110

Harribey Jean-Marie (2011b), « André Orléan, L'empire de la valeur, Refonder l'économie, Paris, Seuil, 2011. ", Revue de la régulation [En ligne], 10 | 2e semestre / Autumn 2011, mis en ligne le 21 décembre 2011, consulté le 28 mars 2018. URL : http://journals.openedition.org/regulation/9483 Harribey Jean-Marie (2013), La Richesse, la valeur et l'inestimable, Fondements d'une critique socioécologique de l'économie capitaliste, Paris, Les Liens qui libèrent. 
Harribey Jean-Marie (2015), « Derrière l'imposture de la "science" économique, qu'y a-t-il ? », Revue du MAUSS permanente, 2015/1, nº 45 ; http://harribey.u-bordeaux4.fr/travaux/valeur/ imposture-sceco.pdf

Harribey Jean-Marie (2016), « Le paradoxe de la monnaie chez Adam Smith », in Oltra Vanessa \& Harribey Jean-Marie (dir.), Les Lumières d'Adam Smith, La philosophie et l'économie en scène, Lormont, Le Bord de l'eau, p. 139-152.

Harribey Jean-Marie (2017a), «Et pourtant ils produisent », Contretemps, janvier, https:// www.contretemps.eu/harribey-reponse-husson-valeur

Harribey Jean-Marie (2017b), « La validation sociale, point de passage entre le travail et la valeur monétaire », Colloque « Monnaie, dette et souveraineté », Amiens, 11-12 décembre, http:// harribey.u-bordeaux4.fr/travaux/valeur/validation-sociale.pdf

Husson Michel (2012), « Le pire de la valeur », Contretemps, n 13, http://hussonet.free.fr/ pirval.pdf

Husson Michel (2016), «Comptabilité nationale et valeur non marchande », Note hussonet, n - 103, 18 octobre, Contretemps ; http://www.contretemps.eu/husson-fonctionnaires-valeur.

Keen Steve (2014), L'Imposture économique, Paris, Les Éditions de l'atelier.

Keynes John Maynard (1969) [1936], Théorie générale de l'emploi, de l'intérêt et de la monnaie, Paris, Payot.

Lordon Frédéric (dir.) (2008), Conflits et pouvoirs dans les institutions du capitalisme, Paris, Presses de la Fondation des sciences politiques.

Lordon Frédéric (2010), Capitalisme, désir et servitude, Marx et Spinoza, Paris, La Fabrique.

Lordon Frédéric (2010/2017), «Le “Nobel”, l'économie et les neurosciences »; https://

blog.mondediplo.net/2017-10-13-Le-Nobel-l-economie-et-les-neurosciences\#nb1.

Marx Karl, Le Capital (Livre I, 1867 ; Livre II, 1885, Livre III, 1894), dans Euvres, t. 1 (1965) et t. 2 (1968), Paris, Gallimard, coll. « Bibliothèque de La Pléiade ».

Mauss Marcel (1991) [1923-1924], « Essai sur le don, Forme et raison de l'échange dans les sociétés archaïques », in Sociologie et anthropologie, 4 éd., Paris, Puf, coll. » Quadrige ».

Minsky Hyman P. (1982), Can «It» Happen Again ? Essay on Instability and Finance, Armonk M.E. Sharpe.

Minsky Hyman P. (2016), Stabiliser une économie instable, Paris, Les Petits matins.

Montalban Matthieu (2012), « De la place de la théorie de la valeur et de la monnaie dans la théorie de la régulation : critique et synthèse », Revue de la régulation [En ligne], 12 | 2e semestre / Autumn 2012, mis en ligne le 19 décembre 2012, consulté le 28 mars 2018. URL : http:// journals.openedition.org/regulation/9797 ; DOI : 10.4000/regulation.9797 Oltra Vanessa \& Harribey Jean-Marie (dir.) (2016), Les Lumières d'Adam Smith, la philosophie et l'économie en scène, Lormont, Le Bord de l'eau.

Orléan André (1999), Le Pouvoir de la finance, Paris, Odile Jacob.

Orléan André (2008), « Monnaie, séparation marchande et rapport salarial », in Lordon Frédéric (dir.), Conflits et pouvoirs dans les institutions du capitalisme, Paris, Presses de la Fondation des sciences politiques, p. 55-87

http://www.pse.ens.fr/orlean/depot/publi/Monnaie0612.pdf. 
Orléan André (2011a), L'Empire de la valeur. Refonder l'économie, Paris, Le Seuil.

Orléan André (2011b), « Réponse à Jean-Marie Harribey », Revue de la régulation [En ligne], 10 | 2e semestre / Autumn 2011, mis en ligne le 21 décembre 2011, consulté le 28 mars 2018. URL : http://journals.openedition.org/regulation/9502

Orléan André (2012), « L'argent fou exalte le théâtre des passions », Le Monde, 20 juillet.

Orléan André (2013), « Le modèle balzacien de la monnaie », in Péraud Alexandre (dir.), La Comédie (in)humaine de l'argent, Lormont, Le Bord de l'eau, p. 129-145.

Orléan André (2015), « La valeur économique comme fait social : la preuve par les évaluations boursières ", Revue de la régulation [En ligne], 18 | 2e semestre / Autumn 2015, mis en ligne le 20 décembre 2015, consulté le 28 mars 2018. URL : http://journals.openedition.org/ regulation/11441; DOI : 10.4000/regulation.11441

Péraud Alexandre (2013), La Comédie (in)humaine de l'argent, Lormont, Le Bord de l'eau.

Piluso Nicolas (2014), « Postulat de la monnaie et théorie de la valeur chez Marx », Revue de la régulation [En ligne], 15 | 1er semestre / Spring 2014, mis en ligne le 27 juin 2014, consulté le 28 mars 2018. URL : http://journals.openedition.org/regulation/10721 ; DOI : 10.4000/ regulation.1072

Piluso Nicolas (2017), « Faut-il abandonner la théorie économique de l'émergence de la monnaie? », Idées, $\mathrm{n}^{\circ}$ 189, septembre 2017, p. 57-61.

Polanyi Karl (1983) [1944], La Grande transformation. Aux origines politiques et économiques de notre temps, Paris, Gallimard.

Popper Karl (1995) [1935], La Logique de la découverte scientifique, Paris, Payot.

Simmel Georg (1999) [1900, 1907], Philosophie de l'argent, Paris, Puf, coll. » Quadrige ».

Spinoza Baruch (2008) [1677], Éthique, Paris, Flammarion, coll. » Le Monde de la philosophie ».

Testart Alain (2001), « Moyen d'échange/moyen de paiement, Des monnaies en général et plus particulièrement des primitives », in Testart Alain (dir.), Aux origines de la monnaie, Paris, Errance, p. 11-60.

Testart Alain (2007), Critique du don. Études sur la circulation non marchande, Paris, Syllepse.

Théret Bruno (dir.) (2007), La Monnaie dévoilée par ses crises, Paris, Éditions de l'EHESS, vol. I, Crises monétaires d'hier et d'aujourd'hui.

Weber Max (1964) [1905], L'Éthique protestante et l'esprit du capitalisme, Paris, Plon-Agora.

Wray Randall (2013), « Money », in G.C. Harcourt and Peter Kriesler (eds), The Oxford Handbook of Post-keynesian Economics, vol. 1, Theory and Origins, Oxford Handbooks Online.

\section{NOTES}

1. Péraud A. (2013), Orléan A. (2013).

2. Voir l'ouvrage collectif Oltra V. \& J.-M. Harribey (dir.) (2016), notamment le chapitre «Le paradoxe de la monnaie chez Adam Smith ».

3. Marx analyse, au début du Capital, le rapport marchand avant même le capital, et Suzanne de Brunhoff (1976, p. 14) indique que «Marx juge nécessaire de commencer l'étude de la monnaie sous sa forme générale non spécifique du mode de production capitaliste ». Cela signifie d'abord que le rapport marchand, donc monétaire, est historiquement antérieur au rapport capitaliste et 
théoriquement plus général que ce dernier. Ensuite, pour notre part, nous en tirons une conséquence que nous ne développerons pas ici : tout rapport marchand est monétaire, mais la réciproque n'est pas vraie, car ce qui est monétaire n'est pas forcément marchand; il s'ensuit qu'il existe un espace pour le développement d'un espace monétaire non marchand dans la société ; voir à ce sujet Harribey J.-M. (2013).

4. Voir Harribey J.-M. (2011a) et (2011b), repris dans Harribey J.-M. (2013), où nous posons la question: la lecture spinoziste de la valeur que font Orléan et Lordon rejoindrait-elle le présupposé néoclassique, selon lequel la valeur naît des «forces désirantes "? Voir la réponse d'Orléan A. (2011b). Voir aussi Husson M. (2012).

5. C'est aussi, la question que pose sans en avoir l'air Laurent Cordonnier (2012) à Orléan.

6. L'auteur poursuit en montrant qu'un taux d'épargne salariale élevé (bas) entraîne une baisse (hausse) des profits et qu'«en cas d'augmentation du taux d'épargne salariale lorsque l'investissement baisse, la diminution des profits s'en trouvera amplifiée ; symétriquement, si le taux d'épargne salariale diminue quand l'investissement augmente, l'augmentation des profits s'en trouvera amplifiée. » (p. 355).

7. Dans le Livre I du Capital, (1965, p. 645).

8. Condillac (1776). Ce qu'il faut remarquer, c'est que Condillac détruit lui-même dans les deux phrases suivantes ce qu'il vient de dire : «Je dis donc que, même sur les bords d'un fleuve, l'eau a une valeur, mais la plus petite possible, parce qu'elle y est infiniment surabondante à nos besoins. Dans un lieu aride, au contraire, elle a une grande valeur; et on l'estime en raison de l'éloignement et de la difficulté de s'en procurer. »

Qu'est-ce donc que la difficulté de s'en procurer sinon le renvoi au coût de production? La théorie de la valeur-utilité s'effondre à peine est-elle formulée. Condillac nous en administre luimême la démonstration quelques lignes plus loin : «Quoiqu'on ne donne point d'argent pour se procurer une chose, elle coûte, si elle coûte un travail. [...] D'après ces réflexions préliminaires, je dis que, lorsque je suis loin de la rivière, l'eau me coûte l'action de l'aller chercher; action qui est un travail, puisqu'elle est faite pour me procurer une chose dont j'ai besoin; et, lorsque je suis sur le bord de la rivière, l'eau me coûte l'action de me baisser pour en prendre ; action qui est un bien petit travail, j'en conviens : c'est moins que le premier coup de bêche. Mais aussi l'eau n'a-telle alors que la plus petite valeur possible.»

https://fr.wikisource.org/wiki/

Le_Commerce_et_le_gouvernement_considérés_relativement_l'un_à_l'autre/Première_Partie/ Section_1

9. Spinoza (2008), III, XXXVII, p. 294.

10. Entendons-nous bien : il y a effectivement perte de «capitalisation boursière ", mais cela ne représente aucune destruction de valeur ou de richesse, simplement destruction de capital fictif.

11. La thèse du don de Mauss ne fait pas l'unanimité chez les anthropologues parce qu'il n'aurait pas suffisamment précisé que faire un don n'ouvre droit à rien d'exigible en contrepartie. C'est notamment la critique portée par Alain Testart (2001 et 2007), qui introduit une distinction à propos de la monnaie entre moyen de paiement et moyen d'échange, le paiement pouvant être séparé d'un échange, comme c'était le cas dans les sociétés primitives. Aujourd'hui encore, selon Testart, le paiement de l'impôt n'est pas un échange ; mais on peut aussi considérer que, en contrepartie de l'impôt, le contribuable a le droit d'exiger que l'on inscrive son enfant à l'école publique.

12. Cela signifie que les sociétés capitalistes concrètes ne sont pas un "copier-coller» de l'idéaltype du capitalisme analysé dans le Livre I du Capital de Marx. Dès lors, la validation par le marché cohabite avec une validation politique des travaux effectués dans la sphère monétaire non marchande. Voir la discussion de la thèse du travail productif au sein de cette sphère, notamment dans la revue Contretemps entre Artous A. (2013 et 2014), Darmangeat C. (2016), Harribey J.-M. (2013 et 2017) et Husson M. (2016). Le dossier se trouve à l'adresse http:// 
harribey.u-bordeaux4.fr/travaux/valeur/index-valeur.html et dans www.contretemps.eu/ dossier-valeur-capitalisme

13. En ce sens, nous ne nous inscrivons pas dans le sillage de la question posée par Nicolas Piluso (2017) : « Faut-il abandonner la théorie économique de l'émergence de la monnaie?»

\section{AUTEUR}

JEAN-MARIE HARRIBEY

Université de Bordeaux, GRETHA-UMR CNRS 5113, jean-marie.harribey@u-bordeaux.fr 